International Journal of Pure and Applied Mathematics

Volume 87 No. 1 2013, 95-114

ISSN: 1311-8080 (printed version); ISSN: 1314-3395 (on-line version)

url: http://www.ijpam.eu

doi: http://dx.doi.org/10.12732/ijpam.v87i1.5

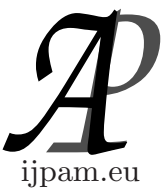

\title{
ANALYSIS ON THE ELLIPTIC SCALAR MULTIPLICATION USING INTEGER SUB-DECOMPOSITION METHOD
}

\author{
Ruma Kareem K. Ajeena ${ }^{1}$, Hailiza Kamarulhaili² \\ ${ }^{1,2}$ School of Mathematical Sciences \\ University Sains Malaysia \\ 11800 USM, Penang, MALAYSIA
}

\begin{abstract}
This study proposes a new approach called, integer sub-decomposition (ISD), to compute any multiple $k P$ of a point $P$ of order $n$ lying on an elliptic curve. Our method depends, in computations, on fast endomorphisms $\psi_{1}$ and $\psi_{2}$ of elliptic curve over prime fields. The integer sub-decomposition to multiple $k P$, when the value of $k$ is decomposed into two values $k_{1}$ and $k_{2}$, where both values or one of them is not bounded by $\pm \mathcal{C} \sqrt{n}$, is illustrated in the following formula:

$$
\begin{aligned}
k P & =k_{11} P+k_{12}\left[\lambda_{1}\right] P+k_{21} P+k_{22}\left[\lambda_{2}\right] P \\
& =k_{11} P+k_{12} \psi_{1}(P)+k_{21} P+k_{22} \psi_{2}(P) .
\end{aligned}
$$
\end{abstract}

where $-\mathcal{C} \sqrt{n}<k_{11}, k_{12}, k_{21}, k_{22}<\mathcal{C} \sqrt{n}$. The integers $k_{11}, k_{12}, k_{21}$ and $k_{22}$ are computed by solving a closest vector problem in lattice. Consequently, as for this sub-decomposition, we have managed to increase the percentage of a successful computation of $k P$. Moreover, the gap in the proof of the bound of kernel $\mathcal{K}$ vectors of the reduction map $T:(a, b) \rightarrow a+\lambda b(\bmod n)$ on ISD method will be filled through the analysis of the multiplier $k$, using two fast endomorphisms with minimal polynomials $X^{2}+r X_{i}+s_{i}$ for $i=1,2,3$. In particular, we prove an integer sub-decomposition (ISD) with explicit constant

$$
k P=k_{11} P+k_{12} \psi_{1}(P)+k_{21} P+k_{22} \psi_{2}(P),
$$

with

$\max \left\{\left|k_{11}\right|,\left|k_{12}\right|\right\}$ and $\max \left\{\left|k_{21}\right|,\left|k_{22}\right|\right\}<\sqrt{1+\left|r_{i}\right|+s_{i}} \sqrt{n}$, for $i=1,2,3$.

Received: $\quad$ May 3, 2013

(c) 2013 Academic Publications, Ltd.

$\S$ Correspondence author url: www.acadpubl.eu 
AMS Subject Classification: 06-xx, 18B35, 06Bxx, 03G10

Key Words: elliptic curves, fast performance, efficiently-computable endomorphisms, integer sub-decomposition

\section{Introduction}

The attractive features of elliptic curves history awarded it studying by mathematicians over a hundred of years to solve a variety of problems. The entry of these curves into cryptography independently by Neal Koblitz [1] and Victor Miller [2] in 1985 who suggested elliptic curve public key cryptosystems. The elliptic curves performance has active importance in the security level as a traditional asymmetric cryptosystem, such as RSA [3],[4]. The fundamental step of elliptic curve cryptosystems is to compute elliptic curve scalar multiplication $k P$ for a point $P$ which has a large prime order $n$. To accomplish this end, various methods have been innovated, adopting on elliptic curves $E$ over finite fields[5],[6],[7] and [8]. A group of methods cleverly employs a distinguished endomorphism $\psi \in \operatorname{End}(E)$ to split a large computation into a sequence of cheaper ones, so that the overall computational cost will be lowered [3].

Recently, Gallant, Lambert and Vanstone [9],[10],[11] used such a technique that, contrary to the previous ones, also applied to curves defined over large prime fields. Their method uses an efficiently computable endomorphism $\psi \in$ $\operatorname{End}(E)$ to rewrite $k P$ as

$$
k P=k_{1} P+k_{2} \psi(P) \text {, with } \max \left\{\left|k_{1}\right|,\left|k_{2}\right|\right\}=O(\sqrt{n}) .
$$

Their key point is an algorithm, that will be called the GLV method, which inputs integers $n$ and $\lambda \in[1, n-1]$ and produces for any $k(\bmod n)$, two residues $k_{1}$ and $k_{2}(\bmod n)$ such that

$$
k=k_{1}+\lambda k_{2}(\bmod n) .
$$

On the other hand, they do not succeed to give an upper bound on $\max \left\{\left|k_{1}\right|\right.$, $\left.\left|k_{2}\right|\right\}$ and they give a guided estimation shows that this must be $O(\sqrt{n})$, but it does not demonstrate any estimation of the concerned constant in their study too. The first appearance for an upper bound was in [12] where a different method was used. Moreover, we were perceived of another usage to the GLV method [11] where a necessary condition is innovated to be sure that the constant in $O(\sqrt{n})$ is 1 in equation (1.1). This algorithm was the alternative to the presented GLV method. 
Improving the GLV algorithm would be to find the decomposition

$$
k P=k_{1} P+k_{2} \psi(P)+\ldots+k_{d} \psi^{d-1}(P) \text {, with } \max \left\{\left|k_{i}\right|\right\}=O\left(n^{\frac{1}{d}}\right) .
$$

In general using the GLV paradigm in equation (1.3) is not possible, since the powers $\psi^{i}$ are independent over $Z$ only when $i<2$. However, a class of $\psi^{\prime} s$ for which such a decomposition exists is found as in [13].

Starting with analyzing the GLV method of Gallant, Lambert and Vanstone, our study uses two fast endomorphisms with minimal polynomials $X^{2}+r_{i} X+$ $s_{i}$, for $i=1,2,3$ to compute any multiple $k P$ of a point $P$ of order $n$ lying on an elliptic curve. When both values or one of them is not bounded by $\pm \sqrt{1+\left|r_{i}\right|+s_{i}} \sqrt{n}, i=1,2,3$, the value $k$ is then decomposed into the values $k_{1}$ and $k_{2}$. The sub-decomposition from $k=k_{1}+k_{2} \lambda(\bmod n)$ is shown clearly as follows:

$$
k_{1}=k_{11}+k_{12} \lambda_{1}(\bmod n) \text { and } k_{2}=k_{21}+k_{22} \lambda_{2}(\bmod n) .
$$

We calculate, in particular, the integer sub-decomposition (ISD) as follows:

$$
\begin{aligned}
k P & =k_{11} P+k_{12}\left[\lambda_{1}\right] P+k_{21} P+k_{22}\left[\lambda_{2}\right] P \\
& =k_{11} P+k_{12} \psi_{1}(P)+k_{21} P+k_{22} \psi_{2}(P) .
\end{aligned}
$$

where $-\sqrt{1+\left|r_{i}\right|+s_{i}} \sqrt{n}<k_{11}, k_{12}, k_{21}, k_{22}<\sqrt{1+\left|r_{i}\right|+s_{i}} \sqrt{n}, i=1,2,3$. A proof is supplied, in this paper, that the ISD algorithm works by producing a required upper bound of the kernel $\mathcal{K}$ vectors of the reduction map $T:(a, b) \rightarrow$ $a+\lambda b(\bmod n)$. We prove, in particular, an integer sub-decomposition with explicit constant

$$
\begin{gathered}
k P=k_{11} P+k_{12} \psi_{1}(P)+k_{21} P+k_{22} \psi_{2}(P), \text { with } \\
\max \left\{\begin{array}{l}
\left\{\left|k_{11}\right|,\left|k_{12}\right|\right\} \\
\left\{\left|k_{21}\right|,\left|k_{22}\right|\right\}
\end{array}\right\}<\sqrt{1+\left|r_{i}\right|+s_{i}} \sqrt{n} \text {, for } i=1,2,3 .
\end{gathered}
$$

The outline of this paper shows: Section 2 gives a summary of the Mathematical background to clarify elliptic curve $E$ over prime field and endomorphisms on it. Section 3 reviews the procedure of scalar multiplication using a GLV method and fills the logical gap of this method. Section 4 shows the value of the bound $\mathcal{C}$ of kernel vectors of the reduction $T$ in GLV method. Section 5 presents a new method called, integer sub-decomposition (ISD), to compute scalar multiplication depending on the sub-decomposition and demonstrates the filling up of the logical gap of the ISD method. Section 6 displays the Mathematical proofs which help us find the value of the bound $\mathcal{C}$ of kernel vectors of the reduction map $T$ on ISD method. Finally, Section 7 draws the concluding remarks. 


\section{Mathematical Background}

\subsection{Elliptic Curves over Prime Fields}

Definition 2.1. Let $p \neq 2,3$. An elliptic curve $E\left(F_{p}\right)$ over $F_{p}$, is defined by an equation of the form [14]:

$$
E: Y^{2}=X^{3}+A X+B(\bmod p),
$$

where $A, B \in F_{p}$. The curve $E$ is said to be non-singular if it has no double zeroes, that means the discriminant $D_{E}=4 A^{3}+27 B^{2} \neq 0(\bmod p)$.

Definition 2.2. Let $E\left(F_{p}\right)$ be an elliptic curve defined in equation (2.1) over the field $F_{p}, P=\left(x_{P}, y_{P}\right)$ and $Q=\left(x_{Q}, y_{Q}\right)$ two points on $E$ such that $P, Q \neq \infty$. We define $P+Q=R=\left(x_{R}, y_{R}\right)$ as follows [14] and [15]:

$$
\begin{gathered}
\mu \equiv\left\{\begin{array}{c}
\left(\frac{y_{Q}-y_{P}}{x_{Q}-x_{P}}\right)(\bmod p), \text { if } P \neq Q \\
\left(\frac{3 x_{P}^{2}+A}{2 y_{p}}\right)(\bmod p), \text { if } P=Q
\end{array}\right. \\
\left\{\begin{array}{l}
x_{R} \equiv \lambda^{2}-x_{P}-x_{Q}(\bmod p) \\
y_{R} \equiv \lambda\left(x_{P}-x_{R}\right)-y_{P}(\bmod p) .
\end{array}\right.
\end{gathered}
$$

A special case when $P=-Q$ then $P+Q=\infty$.

\subsection{Endomorphisms of Elliptic Curve over Prime Fields}

Assume that $E$ is an elliptic curve defined over the finite field $F_{p}$. The point at infinity is denoted by $O_{E}$. The set of $F_{p}$-rational points on $E$ forms the group $E\left(F_{p}\right)$. A rational map $\psi: E \rightarrow E$ satisfies $\psi\left(O_{E}\right)=O_{E}$ dubbed an endomorphism of $E$. The endomorphism $\psi$ will be defined over $F_{q}$ where $q=p^{n}$, if the rational map is defined over $F_{q}$. Therefore, clearly, for any $n \geq 1$, $\psi$ is a group homomorphism of $E\left(F_{p}\right)$ and also of $E\left(F_{q}\right)$ [3] and [15].

Definition 2.3. The endomorphism of elliptic curve $E$ defined over $F_{q}$ is the $m$ - multiplication map $[m]: E \rightarrow E$ defined by

$$
P \rightarrow m P
$$

for each $m \in Z$. The negation map [-1]: $E \rightarrow E$ defined by $P \rightarrow-P$ is a special case from $m$-multiplication map [3]. 
Theorem 2.4. (Hasse Theorem). Let $E$ be an elliptic curve over a finite field $F_{p}[3]$. Then, the order of $E\left(F_{p}\right)$ satisfies

$$
\left|p+1-\# E\left(F_{p}\right)\right| \leq 2 \sqrt{p} .
$$

Definition 2.5. The rectangle norm $[4]$ of $(x, y)$ is defined by $\max \{|x|,|y|\}$. We denote it by $|(x, y)|$.

\section{Bridging the Logical Gaps of the GLV Algorithm}

The Gallant-Lambert-Vanstone's computation method [9] will be briefly summarized in this part. Assume that $F_{q}$ is a finite field. The point $P=(x, y)$ is a point on an elliptic curve $E$ defined over a field $F_{q}$, with order $n$ such that the cofactor $h=\# E\left(F_{q}\right) / n$ is small, say $h \leq 4$. The characteristic polynomial of a non trivial endomorphism $\psi$ defined over $F_{q}$ takes the form $X^{2}+r X+s$, where $r$ and $s$ are actually small fixed integers. By the Hasse bound, since $n$ is large, then $\psi(P)=\lambda P$ for some $\lambda \in[1, n-1]$. As a matter of fact, there is only one copy of $Z / n$ inside $E\left(F_{p}\right)$ and $\psi(P)$ has also an order dividing $n$. Moreover, the parameter $\lambda$ is a root of $X^{2}+r X+s$ modulo $n$, where the case $\lambda=0$ is excluded from all cases.

The definition of the group homomorphism $T$ as follows:

$$
\begin{aligned}
T: & Z \times Z \rightarrow Z / n \\
& (i, j) \rightarrow i+\lambda j(\bmod n)
\end{aligned}
$$

represents a pivotal point in GLV method. Let $\mathcal{K}=\operatorname{ker} T$. Obviously, $\mathcal{K}$ is a sublattice of $Z \times Z$. And let $v_{1}$ and $v_{2}$ be two linearly independent vectors of $\mathcal{K}$ satisfying $\max \left\{\left|v_{1}\right|,\left|v_{2}\right|\right\}<M$ for some $M>0$, where $|\cdot|$ indicates to any metric norm. Consider

$$
(k, 0)=\beta_{1} v_{1}+\beta_{2} v_{2},
$$

where $\beta_{i} \in Q$. Then the rounding of $\beta_{i}$ to the nearest integer is $b_{i}=\left\lfloor\beta_{i}\right\rceil=$ $\left\lfloor\beta_{i}+1 / 2\right\rceil$ and suppose that $v=b_{1} v_{1}+b_{2} v_{2}$. Observe that $v \in \mathcal{K}$ and that $u=(k, 0)-v$ is short. The triangle inequality gives us the following fact

$$
\left|u_{0}\right| \leq\left|\frac{v_{1}+v_{2}}{2}\right|<M .
$$

If one puts

$$
\left(k_{1}, k_{2}\right)=u_{0},
$$


then from equation (1.2), one can have

$$
k P=k_{1} P+k_{2} \psi(P), \text { with }\left|\left(k_{1}, k_{2}\right)\right|<M .
$$

In this way, it is fundamental in the GLV method that $M$ should be as small as possible, taking into consideration that by a simple counting argument we must have $M \geq \sqrt{n} / 2$. Gallant et. al, then, claim without proof the fact that

$$
M \leq \mathcal{C} \sqrt{n}
$$

for some constant $\mathcal{C}[4]$.

\section{A Value for $\mathcal{C}$ in the GLV Algorithm}

Remember that the extended Euclidean algorithm applied to $n$ and $\lambda$ is used by the GLV algorithm to generate a sequence of relations

$$
s_{l} n+t_{l} \lambda=r_{l}, \text { for } l=0,1,2, \ldots,
$$

where $\left|s_{l}\right|<\left|s_{l+1}\right|$ for $l \geq 1,\left|t_{l}\right|<\left|t_{l+1}\right|$ and $r_{l}>r_{l+1} \geq 0$ for $l \geq 0$. Also, we have from Lemma (1-iv) in [9]:

$$
r_{l}\left|t_{l+1}\right|+r_{l+1}\left|t_{l}\right|=n \text { for all } l \geq 0 .
$$

The index $m$ of the GLV algorithm defines as the largest integer for which $r_{m}>\sqrt{n}$. Then (4.2) with $l=m$ gives that $\left|t_{m+1}\right|<\sqrt{n}$, so that the kernel vector $v=\left(r_{m+1},-t_{m+1}\right)$ has rectangle norm bounded by $\sqrt{n}$. The GLV algorithm then sets $v_{2}$ to be the shorter between $\left(r_{m},-t_{m}\right)$ and $\left(r_{m+2},-t_{m+2}\right)$, but does not give any estimate on the size of $v_{2}$. In reality, Gallant et al. claimed that

$$
\min \left(\left|\left(r_{m},-t_{m}\right)\right|\right),\left|\left(r_{m+2},-t_{m+2}\right)\right| \leq \mathcal{C} \sqrt{n} .
$$

This will be explained with an explicit value of $\mathcal{C}$ [4]. Let $\lambda$ and $\mu$ be the zeros of $X^{2}+r X+s(\bmod n)$. For any $(x, y) \in \mathcal{K}-\{(0,0)\}$, one can have $0 \equiv(x+\lambda y)(x+\mu y) \equiv x^{2}-r x y+s y^{2}(\bmod n)$, hence, since $X^{2}+r X+s$ is irreducible in $Z[X]$, one must have $x^{2}-r x y+s y^{2} \geq n$. Certainly, this leads to

$$
\max (|x|,|y|) \geq \sqrt{\frac{n}{1+|r|+s}} .
$$

In particular,

$$
\left|\left(r_{m+1},-t_{m+1}\right)\right| \geq \sqrt{n} / \sqrt{1+|r|+s} .
$$


There are two cases of the components of the vector $v$ :

Case 1.[4] If $\left|t_{m+1}\right| \geq \sqrt{n} / \sqrt{1+|r|+s}$. Then, the equation (4.2) with $l=m$ produces that $r_{m}<\sqrt{1+|r|+s} \sqrt{n}$, hence

$$
\left|\left(r_{m},-t_{m}\right)\right|<\sqrt{1+|r|+s} \sqrt{n} .
$$

Case 2. [4] If $r_{m+1} \geq \sqrt{n} / \sqrt{1+|r|+s}$. The same equation (4.2) with $l=m+1$ implies that $\left|t_{m+2}\right|<\sqrt{1+|r|+s} \sqrt{n}$, hence

$$
\left|\left(r_{m+2},-t_{m+2}\right)\right|<\sqrt{1+|r|+s} \sqrt{n} .
$$

Theorem 4.1. An admissible value [4] for $\mathcal{C}$ is

$$
\mathcal{C}=\sqrt{1+|r|+s}
$$

In particular, the decomposition of any multiple $k P$ can take the form

$$
k P=k_{1} P+k_{2} \psi(P), \text { with } \max \left\{\left|k_{1}\right|,\left|k_{2}\right|\right\}<\sqrt{1+|r|+s} \sqrt{n} .
$$

\section{Bridging the Logical Gaps of the (ISDA) Integer Sub-Decomposition Algorithm}

The integer sub-decomposition computation method can be interpreted through this section as follows. Assume that $F_{q}$ is a finite field. The point $P=(x, y)$ is a point on an elliptic curve $E$ defined over a field $F_{q}$, with order $n$ such that the cofactor $h=\# E\left(F_{q}\right) / n$ is small, say $h \leq 4$. The characteristic polynomials of non trivial endomorphisms $\psi_{1}$ and $\psi_{2}$ defined over $F_{q}$ take the form $X^{2}+$ $r_{i} X+s_{i}$, where $r_{i}$ and $s_{i}$ are actually small fixed integers and $i=1,2,3$. By the Hasse bound, since $n$ is large, then, $\psi_{1}(P)=\lambda_{1} P$ and $\psi_{2}(P)=\lambda_{2} P$ for some $\lambda_{1}$ and $\lambda_{2} \in[1, n-1]$. Actually, there is only one copy of $Z / n$ inside $E\left(F_{q}\right)$ and $\psi_{1}(P)$ and $\psi_{2}(P)$ have also an order dividing $n$. Furthermore, the parameters $\lambda_{j}, j=0,1,2$, are roots of $X^{2}+r_{i} X+s_{i}$ modulo $n, i=1,2,3$ and the cases $\lambda_{1}$ and $\lambda_{2}=0$ are excluded from all cases.

A fundamental role of the ISD method lies in the definition of the group homomorphism

$$
\begin{aligned}
T: & Z \times Z \rightarrow Z / n \\
& (a, b) \rightarrow a+\lambda_{j} b(\bmod n)
\end{aligned}
$$


where $j=0,1,2$. Let $\mathcal{K}=\operatorname{ker} T$. Clearly, the $\mathcal{K}$ is a sublattice $Z \times Z$. Let $v_{1}, v_{2}, v_{3}, v_{4}, v_{5}$ and $v_{6}$ be linearly independent vectors of $\mathcal{K}$ and integer lattice points that satisfy

$$
\max \left\{\begin{array}{l}
\left|v_{1}\right|,\left|v_{2}\right| \\
\left|v_{3}\right|,\left|v_{4}\right| \\
\left|v_{5}\right|,\left|v_{6}\right|
\end{array}\right\}<M
$$

for some $M>0$, where $|\cdot|$ denotes to any metric norm. These points can be computed by solving the closest vector problem in a lattice which is embodied in using a GLV generator algorithm in [3] to compute $\left\{v_{1}, v_{2}\right\}$ and our modified ISD generators algorithm (1) in Appendix (A) to compute $\left\{v_{3}, v_{4}\right\}$ and $\left\{v_{5}, v_{6}\right\}$.

Express

$$
\left\{\begin{array}{l}
(k, 0)=\beta_{1} v_{1}+\beta_{2} v_{2}, \\
\left(k_{1}, 0\right)=\beta_{3} v_{3}+\beta_{4} v_{4}, \\
\left(k_{2}, 0\right)=\beta_{5} v_{5}+\beta_{6} v_{6},
\end{array}\right.
$$

where $\beta_{i} \in Q, i=1,2,3,4,5,6$. Then the rounding of $\beta_{i}$ to the nearest integer $b_{i}=\left\lfloor\beta_{i}\right\rceil=\left\lfloor\beta_{i}+1 / 2\right\rceil$ and let

$$
\left\{\begin{array}{c}
v=b_{1} v_{1}+b_{2} v_{2}, \\
v^{\prime}=b_{3} v_{3}+b_{4} v_{4}, \\
v^{\prime \prime}=b_{5} v_{5}+b_{6} v_{6} .
\end{array}\right.
$$

Observe that $v, v^{\prime}, v^{\prime \prime} \in \mathcal{K}$ and these

$$
\left\{\begin{array}{c}
u_{0}=(k, 0)-v, \\
u_{1}=\left(k_{1}, 0\right)-v^{\prime}, \\
u_{2}=\left(k_{2}, 0\right)-v^{\prime \prime} .
\end{array}\right.
$$

are short. By the triangle inequality, one can obtain

$$
\left\{\begin{array}{l}
\left|u_{0}\right| \leq\left|\frac{v_{1}+v_{2}}{2}\right| \\
\left|u_{1}\right| \leq\left|\frac{v_{3}+v_{4}}{2}\right| \\
\left|u_{2}\right| \leq\left|\frac{v_{5}+v_{6}}{2}\right|
\end{array}\right\}<M .
$$

If one sets

$$
\left(k_{1}, k_{2}\right)=u_{0},
$$

then

$$
k=k_{1}+\left(k_{2} \lambda\right)(\bmod n)
$$


where $k_{1}$ and $k_{2}$ are integers resulting from the decomposition of the multiplier $k$ by using the balanced length-two representation of a multiplier algorithm [3]. The formula in the equation (5.4) is equivalent to

$$
k=k_{1}+k_{2}^{\prime}(\bmod n), \text { with }\left|\left(k_{1}, k_{2}^{\prime}\right)\right|>M .
$$

Thus, the main idea of ISD method is to sub-decompose the values $k_{1}$ and $k_{2}^{\prime}$ when both values or one of them is not bounded by $\pm M$. Therefore, we decompose $k_{1}$ and $k_{2}^{\prime}$ again into integers $k_{11}, k_{12}, k_{21}$ and $k_{22}$ which means that the sub-decomposition of $k$ by applying the modified balanced length-two representation of a sub-decomposition multiplier algorithm (2), in Appendix (B), as follows:

$$
k=k_{11}+k_{12} \lambda_{1}+k_{21}+k_{22} \lambda_{2}(\bmod n)
$$

with $-M<k_{11}, k_{12}, k_{21}, k_{22}<M$ from any ISD generators $\left\{v_{3}, v_{4}\right\}$ and $\left\{v_{5}, v_{6}\right\}$. Assume that one puts

$$
u_{1}=\left(k_{11}, k_{12}\right) \text { and } u_{2}=\left(k_{21}, k_{22}\right),
$$

then

$$
k_{1}=k_{11}+k_{12} \lambda_{1}(\bmod n) \text { and } k_{2}=k_{21}+k_{22} \lambda_{2}(\bmod n)
$$

which are equivalent to

$$
k_{1} P=k_{11} P+k_{12} \psi_{1}(P) \text { and } k_{2} P=k_{21} P+k_{22} \psi_{2}(P) .
$$

That means

$$
k P=k_{11} P+k_{12} \psi_{1}(P)+k_{21} P+k_{22} \psi_{2}(P),
$$

with

$$
\left|\left(k_{11}, k_{12}\right)\right| \text { and }\left|\left(k_{21}, k_{22}\right)\right|<M .
$$

The fast performance of scalar multiplication $k P$ in equation (5.11) determines our modification, in algorithm (3), in Appendix (C), that uses in computations two endomorphisms $\psi_{1}(P)=\left[\lambda_{1}\right] P$ and $\psi_{2}(P)=\left[\lambda_{2}\right] P$, where $P \in E\left(F_{p}\right), \lambda_{1}, \lambda_{2} \in[1, n-1]$ and $\lambda_{1} \neq \pm \lambda_{2}$. Basically, $M$ is as small as possible in the ISD method and we must have $M \geq \sqrt{n} / 2$. The integer subdecomposition method, ISD will help increase $50 \%$ more successful rate as compared to the GLV method in the computation of the $k P$. See algorithm (4) in Appendix (D). 


\section{A Value for $\mathcal{C}$ in an Integer Subdecomposition Method (ISDM)}

In this section, we overcome on the omission which applied to ISD method that focuses on the sub-decomposition of integer $k$ when the values were decomposed $k_{1}$ and $k_{2}$ are not bounded by $\pm M$. The using of the extended Euclidean algorithm in the ISD algorithm utilized to $n$ and $\lambda_{0}$ firstly to generate a sequence of relations in the equation (4.1). Also, we had the condition in equation (4.2) from Lemma (1-iv) in [9]. The GLV algorithm used in ISD method defines the index $m$ as the largest integer for which $r_{m}>\sqrt{n}$. Then, the equation (4.2) with $l=m$ gives that $\left|t_{m+1}\right|<\sqrt{n}$, so that the vector $v_{1}=\left(r_{m+1},-t_{m+1}\right)$ in $\mathcal{K}$, has a rectangle norm bounded by $M$. The modified GLV algorithm, then, sets $v_{2}$ to be the shorter between $\left(r_{m},-t_{m}\right)$ and $\left(r_{m+2},-t_{m+2}\right)$ and satisfies the conditions in Lemmas (1) and (2) in [11] such that

$$
\min \left(\left|\left(r_{m},-t_{m}\right)\right|,\left|\left(r_{m+2},-t_{m+2}\right)\right|\right) \leq \mathcal{C} \sqrt{n},
$$

where $\operatorname{gcd}\left(r_{m},-t_{m}\right)=1$ and $\operatorname{gcd}\left(r_{m+2},-t_{m+2}\right)=1$, with an explicit value of $\mathcal{C}=$ 1.

In similar way, we can set the vectors $v_{4}$ and $v_{6}$ by depending on $v_{3}$ and $v_{5}$ as follows

$$
\min \left\{\begin{array}{l}
\left|\left(\bar{r}_{m},-\bar{t}_{m}\right)\right|,\left|\left(\bar{r}_{m+2},-\bar{t}_{m+2}\right)\right| \\
\left|\left(\hat{r}_{m},-\hat{t}_{m}\right)\right|,\left|\left(\hat{r}_{m+2},-\hat{t}_{m+2}\right)\right|
\end{array}\right\} \leq \mathcal{C} \sqrt{n}
$$

where

$$
\operatorname{gcd}\left\{\begin{array}{c}
\left(\bar{r}_{m},-\bar{t}_{m}\right) \\
\left(\bar{r}_{m+2},-\bar{t}_{m+2}\right) \\
\left(\hat{r}_{m},-\hat{t}_{m}\right) \\
\left(\hat{r}_{m+2},-\hat{t}_{m+2}\right)
\end{array}\right\}=1
$$

with an explicit value $\mathcal{C}=1$.

Now, one can show the explicit value of $\mathcal{C}$ when this value greater than 1 as follows. Let $\lambda_{j}$ and $\mu_{j} \in[1, n-1], j=0,1,2$, be the zeros of $X^{2}+r_{i} X+$ $s_{i}(\bmod n), i=1,2,3$. For any $(x, y) \in \mathcal{K}-\{(0,0)\}$, then

$$
0 \equiv\left(x+\lambda_{j} y\right)\left(x+\mu_{j} y\right) \equiv x^{2}-r_{i} x y+s_{i} y^{2}(\bmod n),
$$

hence, since $X^{2}+r_{i} X+s_{i}$ is irreducible in $Z[X]$, one must have

$$
x^{2}-r_{i} x y+s_{i} y^{2} \geq n .
$$

This certainly leads to

$$
\max (|x|,|y|) \geq \sqrt{\frac{n}{1+\left|r_{i}\right|+s_{i}}}, i=1,2,3 .
$$


In particular,

$$
\left\{\begin{array}{l}
\left|\left(r_{m+1},-t_{m+1}\right)\right| \\
\left|\left(\bar{r}_{m+1},-\bar{t}_{m+1}\right)\right| \\
\left|\left(\hat{r}_{m+1},-\hat{t}_{m+1}\right)\right|
\end{array}\right\} \geq \sqrt{n} / \sqrt{1+\left|r_{i}\right|+s_{i}}, \text { where } i=1,2,3 .
$$

Theorem 6.1. Suppose that

$$
\left\{\begin{array}{l}
\left|t_{m+1}\right| \\
\left|\bar{t}_{m+1}\right| \\
\left|\hat{t}_{m+1}\right|
\end{array}\right\} \geq \sqrt{n} / \sqrt{1+\left|r_{i}\right|+s_{i}}, \text { where } i=1,2,3 .
$$

Then, the equation (4.2) with $l=m$ implies that

$$
\left\{\begin{array}{l}
r_{m} \\
\bar{r}_{m} \\
\hat{r}_{m}
\end{array}\right\} \geq \sqrt{n} / \sqrt{1+\left|r_{i}\right|+s_{i}}, \text { where } i=1,2,3
$$

hence,

$$
\left\{\begin{array}{l}
\left|\left(r_{m},-t_{m}\right)\right| \\
\left|\left(\bar{r}_{m},-\bar{t}_{m}\right)\right| \\
\left|\left(\hat{r}_{m},-\hat{t}_{m}\right)\right|
\end{array}\right\} \geq \sqrt{n} / \sqrt{1+\left|r_{i}\right|+s_{i}}, \text { where } i=1,2,3 .
$$

Proof. From the conditions in equation (4.1) $\left|t_{l}\right|<\left|t_{l+1}\right|, r_{l}>r_{l+1} \geq 0$ and in equation (4.2), $r_{l}\left|t_{l+1}\right|+r_{l+1}\left|t_{l}\right|=n$ for all $l \geq 0$.

$\Rightarrow n=r_{l}\left|t_{l+1}\right|+r_{l+1}\left|t_{l}\right|>r_{l}\left|t_{l+1}\right|+r_{l}\left|t_{l}\right|=r_{l}\left(\left|t_{l+1}\right|+\left|t_{l}\right|\right)$.

That is, $n>r_{l}\left(\left|t_{l+1}\right|+\left|t_{l}\right|\right)$. Since $\left|t_{l+1}\right|>\left|t_{l}\right|$

$\Rightarrow n=r_{l}\left(\left|t_{l+1}\right|+\left|t_{l}\right|\right)=2 r_{l}\left|t_{l+1}\right|$

$\Rightarrow \frac{n}{2}>r_{l}\left(\left|t_{l+1}\right|\right.$. From the hypothesis $\left|t_{m+1}\right| \geq \sqrt{n} / \sqrt{1+\left|r_{i}\right|+s_{i}}, i=1,2,3$,

$\Rightarrow \frac{n}{2}>r_{l} \frac{\sqrt{n}}{\sqrt{1+\left|r_{i}\right|+s_{i}}}$

$\Rightarrow \frac{n \sqrt{1+\left|r_{i}\right|+s_{i}}}{2 \sqrt{n}}>r_{i}$

$\Rightarrow \frac{\sqrt{n} \sqrt{1+\left|r_{i}\right|+s_{i}}}{2}>r_{i}$

$\Rightarrow r_{i}<\frac{\sqrt{n} \sqrt{1+\left|r_{i}\right|+s_{i}}}{2}<\sqrt{n} \sqrt{1+\left|r_{i}\right|+s_{i}}$,

hence,

$$
\left|\left(r_{m},-t_{m}\right)\right|<\sqrt{1+\left|r_{i}\right|+s_{i}} \sqrt{n} \text {, when } i=1 .
$$

In the same way, we can find

$$
\left\{\begin{array}{l}
\left|\left(\bar{r}_{m},-\bar{t}_{m}\right)\right| \\
\left|\left(\hat{r}_{m},-\hat{t}_{m}\right)\right|
\end{array}\right\}<\sqrt{1+\left|r_{i}\right|+s_{i}} \sqrt{n}, \text { where } i=2,3
$$


Theorem 6.2. Assume that

$$
\left\{\begin{array}{l}
r_{m+1} \\
\bar{r}_{m+1} \\
\hat{r}_{m+1}
\end{array}\right\} \geq \sqrt{n} / \sqrt{1+\left|r_{i}\right|+s_{i}}, i=1,2,3 .
$$

The same equation (4.2) with $l=m+1$ implies that

$$
\left\{\begin{array}{c}
\left|t_{m+2}\right| \\
\left|\bar{t}_{m+2}\right| \\
\left|\hat{t}_{m+2}\right|
\end{array}\right\}<\sqrt{1+\left|r_{i}\right|+s_{i}} \sqrt{n}, i=1,2,3 .
$$

hence,

$$
\left\{\begin{array}{l}
\left|\left(r_{m+2},-t_{m+2}\right)\right| \\
\left|\left(\bar{r}_{m+2},-\bar{t}_{m+2}\right)\right| \\
\left|\left(\hat{r}_{m+2},-\hat{t}_{m+2}\right)\right|
\end{array}\right\}<\sqrt{1+\left|r_{i}\right|+s_{i}} \sqrt{n}, i=1,2,3 .
$$

Proof. From the conditions in equation (4.1) $\left|t_{l}\right|<\left|t_{l+1}\right|, r_{l}>r_{l+1} \geq 0$ and in equation (4.2), $r_{l}\left|t_{l+1}\right|+r_{l+1}\left|t_{l}\right|=n$ for all $l \geq 0$.

$\Rightarrow n=r_{l}\left|t_{l+1}\right|+r_{l+1}\left|t_{l}\right|>r_{l}\left|t_{l+1}\right|+r_{l+1}\left|t_{l+1}\right|=\left|t_{l+1}\right|\left(r_{l}+r_{l+1}\right)$.

That is, $n>\left|t_{l+1}\right|\left(r_{l}+r_{l+1}\right)$. Since $r_{l}>r_{l+1} \geq 0$,

$\Rightarrow n>\left|t_{l+1}\right|\left(r_{l}+r_{l+1}\right)=2 r_{l+1}\left|t_{l+1}\right|$.

$\Rightarrow \frac{n}{2}>r_{l+1}\left|t_{l+1}\right|$. From the hypothesis $r_{m+1} \geq \sqrt{n} / \sqrt{1+\left|r_{i}\right|+s_{i}}, i=1,2,3$.

$\Rightarrow \frac{n}{2}>\frac{\sqrt{n}}{\sqrt{1+\left|r_{i}\right|+s_{i}}}\left|t_{l+1}\right|$,

$\Rightarrow \frac{\sqrt{1+\left|r_{i}\right|+s_{i}} \sqrt{n}}{2}>\left|t_{l+1}\right|$,

$\Rightarrow\left|t_{l+1}\right|<\frac{\sqrt{1+\left|r_{i}\right|+s_{i}} \sqrt{n}}{2}<\sqrt{1+\left|r_{i}\right|+s_{i}} \sqrt{n}$. Since $l=m+1$,

$\Rightarrow\left|t_{l+2}\right|<\sqrt{1+\left|r_{i}\right|+s_{i}} \sqrt{n}, i=1$.

In similar way, we can prove

$$
\left\{\begin{array}{l}
\left|\left(\bar{r}_{m+2},-\bar{t}_{m+2}\right)\right| \\
\left|\left(\hat{r}_{m+2},-\hat{t}_{m+2}\right)\right|
\end{array}\right\}<\sqrt{1+\left|r_{i}\right|+s_{i}} \sqrt{n}, i=2,3 .
$$

Hence,

$$
\left\{\begin{array}{l}
\left|\left(r_{m+2},-t_{m+2}\right)\right| \\
\left|\left(\bar{r}_{m+2},-\bar{t}_{m+2}\right)\right| \\
\left|\left(\hat{r}_{m+2},-\hat{t}_{m+2}\right)\right|
\end{array}\right\}<\sqrt{1+\left|r_{i}\right|+s_{i}} \sqrt{n}, i=1,2,3
$$

Theorem 6.3. An admissible value for $\mathcal{C}$ is

$$
\mathcal{C}=\sqrt{1+\left|r_{i}\right|+s_{i}}, i=1,2,3 .
$$


In particular, any multiple $k P$ can be decomposed as in equation (5.10) with

$$
\max \left\{\begin{aligned}
\left\{\left|k_{1}\right|,\left|k_{2}\right|\right\} & <\sqrt{1+\left|r_{1}\right|+s_{1}} \sqrt{n} \\
\left\{\left|k_{11}\right|,\left|k_{12}\right|\right\} & <\sqrt{1+\left|r_{2}\right|+s_{2}} \sqrt{n} \\
\left\{\left|k_{21}\right|,\left|k_{22}\right|\right\} & <\sqrt{1+\left|r_{3}\right|+s_{3}} \sqrt{n} .
\end{aligned}\right.
$$

Proof. First, we want to prove $\mathcal{C}=\sqrt{1+\left|r_{i}\right|+s_{i}}$, for $i=1,2,3$.

From Theorem (6.1), we can obtain

$$
\left\{\begin{array}{l}
\left|\left(r_{m},-t_{m}\right)\right| \\
\left|\left(\bar{r}_{m},-\bar{t}_{m}\right)\right| \\
\left|\left(\hat{r}_{m},-\hat{t}_{m}\right)\right|
\end{array}\right\}<\sqrt{1+\left|r_{i}\right|+s_{i}} \sqrt{n}, \text { for } i=1,2,3
$$

And from Theorem(6.2), we can get

$$
\left\{\begin{array}{l}
\left|\left(r_{m+2},-t_{m+2}\right)\right| \\
\left|\left(\bar{r}_{m+2},-\bar{t}_{m+2}\right)\right| \\
\left|\left(\hat{r}_{m+2},-\hat{t}_{m+2}\right)\right|
\end{array}\right\}<\sqrt{1+\left|r_{i}\right|+s_{i}} \sqrt{n}, i=1,2,3
$$

then

$$
\min \left\{\begin{array}{l}
\left|\left(r_{m},-t_{m}\right),\left(r_{m+2},-t_{m+2}\right)\right| \\
\left|\left(\bar{r}_{m},-\bar{t}_{m}\right),\left(\bar{r}_{m+2},-\bar{t}_{m+2}\right)\right| \\
\left|\left(\hat{r}_{m},-\hat{t}_{m}\right),\left(\hat{r}_{m+2},-\hat{t}_{m+2}\right)\right|
\end{array}\right\}<\sqrt{1+\left|r_{i}\right|+s_{i}} \sqrt{n}, i=1,2,3 .
$$

By comparison between two equations (6.1) and (6.10), we can find the value of $\mathcal{C}$ as in equation (6.8).

Now to prove any multiple $k P$ can be decomposed as in equation (5.10) with the conditions in equation (6.9). Since $X^{2}+r_{i} X+s_{i}$ are irreducible in $Z[X]$, we must have the inequality in equation (6.3). This implies that the inequality in equation (6.4). In particular,

$$
\left\{\begin{array}{l}
\left|\left(r_{m+1},-t_{m+1}\right)\right| \\
\left|\left(\bar{r}_{m+1},-\bar{t}_{m+1}\right)\right| \\
\left|\left(\hat{r}_{m+1},-\hat{t}_{m+1}\right)\right|
\end{array}\right\} \geq \sqrt{n} / \sqrt{1+\left|r_{i}\right|+s_{i}}, \text { for } i=1,2,3,
$$

and $\left|\left(r_{m+1},-t_{m+1}\right)\right|=\left|v_{1}\right|,\left|\left(\bar{r}_{m+1},-\bar{t}_{m+1}\right)\right|=\left|v_{2}\right|$ and $\left|\left(\hat{r}_{m+1},-\hat{t}_{m+1}\right)\right|=\left|v_{3}\right|$. Since $u_{1}=\left(k_{11}, k_{12}\right)$ and $u_{2}=\left(k_{21}, k_{22}\right)$ from equation (5.7) and from equation (5.8), respectively, we can get $k_{1}=k_{11}+k_{12} \lambda_{1}(\bmod n)$ and $k_{2}=k_{21}+$ $k_{22} \lambda_{2}(\bmod n)$ which are equivalent to $k_{1} P=k_{11} P+k_{12} \psi_{1}(P)$ and $k_{2}=$ $k_{21} P+k_{22} \psi_{2}(P)$ as shown in equation(5.9). 
From inequalities in equation (5.2) as

$$
\left|u_{1}\right| \leq\left|\frac{v_{3}+v_{4}}{2}\right|<M \text { and }\left|u_{2}\right| \leq\left|\frac{v_{5}+v_{6}}{2}\right|<M
$$

then

$$
\left|\left(k_{11}, k_{12}\right)\right|<M \text { and }\left|\left(k_{21}, k_{22}\right)\right|<M \text {. }
$$

Since $M \leq \mathcal{C} \sqrt{n}$, then $\left|\left(k_{11}, k_{12}\right)\right|<\mathcal{C} \sqrt{n}$ and $\left|\left(k_{21}, k_{22}\right)\right|<\mathcal{C} \sqrt{n}$. Now, from definition (2.5) of rectangle norm

$$
\left|\left(k_{11}, k_{12}\right)\right|=\max \left(\left|k_{11}\right|,\left|k_{12}\right|\right) \text { and }\left|\left(k_{21}, k_{22}\right)\right|=\max \left(\left|k_{21}\right|,\left|k_{22}\right|\right) .
$$

This means that $\max \left(\left|k_{11}\right|,\left|k_{12}\right|\right)<\mathcal{C} \sqrt{n}$ and $\max \left(\left|k_{21}\right|,\left|k_{22}\right|\right)<\mathcal{C} \sqrt{n}$.

Finally, from equation (6.8) to compute $\mathcal{C}$, we can find

$$
\max \left\{\begin{array}{l}
\left|k_{11}\right|,\left|k_{12}\right| \\
\left|k_{21}\right|,\left|k_{22}\right|
\end{array}\right\}<\sqrt{1+\left|r_{i}\right|+s_{i}} \sqrt{n} \text { for } i=2,3 .
$$

\section{Conclusion}

The present work proposes a new method which help facilitate the use of Gallant et al.'s (GLV) integers are not bounded by $\pm \sqrt{n}$. This new method, namely, the integer sub-decomposition method, ISD will help increase $50 \%$ more successful rate as compared to the GLV method in the computation of the $k P$. This study also, focuses on presenting an accurate analysis of the ISD method that optimizes and proves on existing bound. This bound determines value $\mathcal{C}$ which is greater than 1 , say $\mathcal{C}=\sqrt{1+\left|r_{i}\right|+s_{i}}, i=1,2,3$ in case in which the endomorphism rings $E n d[\psi]$ over $Z$. This analysis can be applied when embedding endomorphism rings $\operatorname{End}[\psi]$ into complex number field $C$, one can further notice that dealing with similar case where $\mathcal{C}>1$ is more complicated than in case in which the endomorphism rings $\operatorname{End}[\psi]$ over $Z$. Moreover, the generalization can include the hyperelliptic curves of the ISD method.

\section{References}

[1] N. Koblitz, Elliptic curve cryptosystems, Mathematics of computation, 48 (1987), 203-209, doi: 10.2307/2007884. 
[2] V. Miller, Use of elliptic curves in cryptography, In: Advances in Cryptology-CRYPTO'85 Proceedings (1986), 417-426, doi: 10.1007/3-54039799-X.

[3] D. Hankerson, A. J. Menezes, and S. Vanstone, Guide to elliptic curve cryptography, Springer Verlag, USA (2004).

[4] F. Sica, M. Ciet, and J.-J. Quisquater, Analysis of the Gallant-LambertVanstone method based on efficient endomorphisms: Elliptic and hyperelliptic curves, In: Selected areas in cryptography (2003), 21-36.

[5] R. Shi and J. Cheng, Two new fast methods for simultaneous scalar multiplication in elliptic curve cryptosystems, In: Networking and Mobile Computing, Springer (2005), 462-470.

[6] C. Negre, Scalar multiplication on elliptic curves defined over fields of small odd characteristic, In: Progress in Cryptology-INDOCRYPT 2005, Springer (2005), 389-402.

[7] R. Barua, S. K. Pandey, and R. Pankaj, Efficient window-based scalar multiplication on elliptic curves using double-base number system, In: Progress in Cryptology-INDOCRYPT 2007, Springer (2007), 351-360.

[8] D. Liu, Z. Tan, and Y. Dai, New elliptic curve multi-scalar multiplication algorithm for a pair of integers to resist SPA, In: Information Security and Cryptology (2009), 253-264.

[9] R. Gallant, R. Lambert, and L. Vanstone, Faster point multiplication on elliptic curves with efficient endomorphisms, In: Advances in CryptologyCRYPTO 2001 (2001), 190-201.

[10] M. Ciet, J.-J. Quisquater, and F. Sica, Preventing differential analysis in GLV elliptic curve scalar multiplication, Cryptographic Hardware and Embedded Systems-CHES 2002 (2003), 1-13.

[11] D. Kim, S. Lim, Integer decomposition for fast scalar multiplication on elliptic curves, In: Selected Areas in Cryptography, Springer (2003), 13-20.

[12] Y.-H. Park, S. Jeong, C. H. Kim, and J. Lim, An alternate decomposition of an integer for faster point multiplication on certain elliptic curves, In: Public Key Cryptography (2002), 323-334. 
[13] V. Muller, Efficient point multiplication for elliptic curves over special optimal extension fields, In: Public-Key Cryptography and Computational Number Theory: Proceedings of the International Conference organized by the Stefan Banach International Mathematical Center Warsaw, Poland, September 11-15, 2000 (2001), p.197.

[14] D. Venturi, Lecture Notes on Algorithmic Number Theory (2000).

[15] L. C. Washington, Elliptic curves: number theory and cryptography, Chapman \& Hall/CRC, USA (2008).

\section{Appendix A. ISD Generators Algorithm}

Algorithm 1 (Find ISD generators $v_{1}=(a, b), v_{2}=(c, d), v_{3}=(g, j)$ and $v_{4}=(e, f)$ for given $n$ and $\lambda_{1}, \lambda_{2} \in Z$, where $\left.\lambda_{1} \neq \pm \lambda_{2}\right)$.

Input. Integers $n, \lambda_{1}, \lambda_{2}$.

Output. The vectors $v_{1}, v_{2}, v_{3}$ and $v_{4}$.

Step 1. Compute $v_{1}=\left(a_{m+1},-b_{m+1}\right)$ and $v_{3}=\left(g_{m+1},-j_{m+1}\right)$ such that $s_{m+1} n+b_{m+1} \lambda_{1}=a_{m+1}$ and $u_{m+1} n+j_{m+1} \lambda_{1}=g_{m+1}$ where $\left|a_{m+1}\right|,\left|b_{m+1}\right|$, $\left|g_{m+1}\right|$ and $\left|j_{m+1}\right|<\mathcal{C} \sqrt{n}$ by using the extended Euclidean algorithm to find firstly the greatest common divisor of $n$ and $\lambda_{1}$ and secondly of the same $n$ and $\lambda_{2}$. (This is the extension of Gallant et al.'s algorithm for two vectors $v_{1}$ and $\left.v_{3}\right)$.

Step 2. Check if each component of $v_{2}$ either $\left(a_{m},-b_{m}\right)$ or $\left(a_{m+2},-b_{m+2}\right)$ and $\left(g_{m},-j_{m}\right)$ or $\left(g_{m+2},-j_{m+2}\right)$ is bounded by $\mathcal{C} \sqrt{n}$, stop and set the shorter of $\left(a_{m},-b_{m}\right)$ and $\left(a_{m+2},-b_{m+2}\right)$ as the second vector $v_{2}$, also set the shorter of $\left(g_{m},-j_{m}\right)$ and $\left(g_{m+2},-j_{m+2}\right)$ as the fourth vector $v_{4}$. Otherwise, go to step 3 .

Step 3. Find any $d^{\prime}, w^{\prime}, f^{\prime}$ and $v^{\prime}$ such that $s_{m+1} d^{\prime}-b_{m+1} w^{\prime}=1$ and $u_{m+1} f^{\prime}-$ $j_{m+1} v^{\prime}=1$.

For example, $d^{\prime}$ and $w^{\prime}$ are obtained from the extended Euclidean algorithm, since $s_{m+1}$ is relatively prime to $-b_{m+1}$, and the same thing with $f^{\prime}$ and $v^{\prime}$ are obtained from the extended Euclidean algorithm, since $u_{m+1}$ is relatively prime to $-j_{m+1}$.

Step 4. Compute

$$
I_{11}=-\frac{d^{\prime}}{b}-\frac{\sqrt{n}}{b}, I_{12}=-\frac{d^{\prime}}{b}+\frac{\sqrt{n}}{b}
$$


and

$$
I_{11}^{\prime}=-\frac{f^{\prime}}{j}-\frac{\sqrt{n}}{j}, I_{12}^{\prime}=-\frac{f^{\prime}}{j}+\frac{\sqrt{n}}{j} .
$$

Step 5. Let

$$
I_{1}=\left[I_{11}, I_{12}\right], \quad I_{1}^{\prime}=\left[I_{11}^{\prime}, I_{12}^{\prime}\right], \text { if } b>0,
$$

and

$$
I_{1}=\left[I_{12}, I_{11}\right], \quad I_{1}^{\prime}=\left[I_{12}^{\prime}, I_{11}^{\prime}\right], \text { if } b<0 .
$$

Step 6. Compute

$$
I_{21}=-\frac{d^{\prime} \lambda_{1}-w^{\prime} n}{a}-\frac{\sqrt{n}}{a}, I_{22}=-\frac{d^{\prime} \lambda_{1}-w^{\prime} n}{a}+\frac{\sqrt{n}}{a} .
$$

Also,

$$
I_{21}^{\prime}=-\frac{f^{\prime} \lambda_{2}-v^{\prime} n}{g}-\frac{\sqrt{n}}{g}, I_{22}^{\prime}=-\frac{f^{\prime} \lambda_{2}-v^{\prime} n}{g}+\frac{\sqrt{n}}{g} .
$$

Step 7. Let $I_{2}=\left[I_{21}, I_{22}\right]$ and $I_{2}^{\prime}=\left[I_{21}^{\prime}, I_{22}^{\prime}\right]$.

Step 8. Find all integers in the intersection of $I_{1}$ and $I_{2}$ and define them by $\alpha_{1}$, also all integers in the intersection of $I_{1}^{\prime}$ and $I_{2}^{\prime}$ and define them by $\alpha_{2}$. Note that the numbers of $\alpha_{1}^{\prime} s$ and $\alpha_{2}^{\prime} s$ are at most 4 . If there is not any of such integers exist, stop.

Step 9. Set $v_{2}=(c, d)$ and $v_{4}=(e, f)$, where

$$
c=w^{\prime} n-d^{\prime} \lambda_{1}+\alpha_{1} a, \quad d=d^{\prime}+\alpha_{1} b
$$

and

$$
e=v^{\prime} n-f^{\prime} \lambda_{2}+\alpha_{2} g, \quad f=f^{\prime}+\alpha_{2} j .
$$

One can easily verify that $v_{2}=(c, d)$ and $v_{4}=(e, f)$ are in the $\mathcal{K}$ and $|c|,|d|,|e|$ and $|f|<\mathcal{C} \sqrt{n}$, therefore, $\left\{v_{1}, v_{2}\right\}$ and $\left\{v_{3}, v_{4}\right\}$ are ISD generators.

\section{Appendix B. Balanced Length-Two Representation of a Sub-Decomposition Multiplier Algorithm}

Algorithm 2 (Balanced length-two representation of a sub-decomposition multiplier algorithm).

Input. Integers $n, \lambda_{1}, \lambda_{2} \in[1, n-1]$, where $\lambda_{1} \neq \pm \lambda_{2}$ and $k_{1}, k_{2} \in[1, n-1]$. Output. Integers $k_{11}, k_{12}, k_{21}$ and $k_{22}$ such that $k=k_{11}+k_{12} \lambda_{1}+k_{21}+$ $k_{22} \lambda_{2}(\bmod n)$ and $\left|k_{11}\right|,\left|k_{12}\right|,\left|k_{21}\right|,\left|k_{22}\right|<\mathcal{C} \sqrt{n}$. 
Step 1. Run ISD generators algorithm (1) with inputs $n, \lambda_{1}$ and $\lambda_{2}$. The algorithm produces the ISD generators $\left\{v_{3}, v_{4}\right\}$ and $\left\{v_{5}, v_{6}\right\}$.

Step 2. Set $v_{3}=\left(\bar{r}_{m+1},-\bar{t}_{m+1}\right)=(\bar{r},-\bar{t})$ and $v_{5}=\left(\hat{r}_{m+1},-\hat{t}_{m+1}\right)=(\hat{r},-\hat{t})$.

Step 3. If $\left(\bar{r}_{m}^{2}+\bar{t}_{m}^{2}\right) \leq\left(\bar{r}_{m+2}^{2}+\bar{t}_{m+2}^{2}\right)$ then set

$$
v_{4}=(\bar{u}, \bar{v}) \leftarrow\left(\bar{r}_{m},-\bar{t}_{m}\right) \text { and } v_{6}=(\hat{u}, \hat{v}) \leftarrow\left(\hat{r}_{m},-\hat{t}_{m}\right)
$$

Else

$$
v_{4}=(\bar{u}, \bar{v}) \leftarrow\left(\bar{r}_{m+2},-\bar{t}_{m+2}\right) \text { and } v_{6}=(\hat{u}, \hat{v}) \leftarrow\left(\hat{r}_{m+2},-\hat{t}_{m+2}\right)
$$

Step 4. Compute $c_{3}=\left\lfloor\bar{v} k_{1} / n\right\rceil, c_{4}=\left\lfloor-\bar{t} k_{1} / n\right\rceil$ and $c_{5}=\left\lfloor\hat{v} k_{2} / n\right\rceil, \quad c_{6}=$ $\left\lfloor-\hat{t} k_{2} / n\right\rceil$.

Step 5. Compute $k_{11}=k_{1}-c_{3} \bar{r}-c_{4} \bar{u}, k_{12}=-c_{3} \bar{t}-c_{4} \bar{v}$ and $k_{21}=k_{2}-c_{5} \hat{r}-$ $c_{6} \hat{u}, \quad k_{22}=-c_{5} \hat{t}-c_{6} \hat{v}$.

Step 6. Return $k_{11}, k_{12}, k_{21}$ and $k_{22}$.

\section{Appendix C. Modification of Point Multiplication with Two Efficiently Computable Endomorphisms Algorithm}

Algorithm 3 (Modification of point multiplication with two efficiently computable endomorphisms algorithm.

Input. Integer $n, k_{1}, k_{2} \in[1, n-1], P \in E\left(F_{p}\right)$, window widths $w_{1}, w_{2}, w_{3}$ and $w_{4}, \lambda_{1}, \lambda_{2} \in Z$, where $\lambda_{1} \neq \pm \lambda_{2}$.

Output. $k P$.

Step 1. Use balanced length-two representation a sub-decomposing of a multiplier algorithm to find $k_{11}, k_{12}, k_{21}$ and $k_{22}$ such that

$$
k=k_{11}+k_{12} \lambda_{1}+k_{21}+k_{22} \lambda_{2}(\bmod n) .
$$

Step 2. Calculate $P_{2}=\psi_{1}(P), P_{3}=\psi_{2}(P)$ and let $P_{1}=P$.

Step 3. Use computing width-w NAF of positive integer algorithm to compute $N A F_{w_{j}}\left(\left|k_{z, j}\right|\right)=\Sigma_{i=1}^{l_{j}-1} k_{z, j, i} 2^{i}$ for $j=1,2$ and $z=1,2$.

Step 4. Let $l_{z}=\max \left\{l_{z, 1}, l_{z, 2}\right\}, z=1,2$.

Step 5. If $k_{z, j}<0$, then set $G_{z, j, i} \leftarrow-G_{z, j, i}$ for $i=0: l_{z}, j=1,2$ and $z=1,2$. 
Step 6. Compute $i P_{j}$ and $i P_{s}$ for $i \in\left\{1,3, \ldots, 2^{w_{j}-1}-1\right\}$ and $i \in\left\{1,3, \ldots, 2^{w_{s}-1}-\right.$ $1\}$, where $j=1,2$ and $s=1,3$.

Step 7. $Q \leftarrow \infty$.

Step 8. For $i=l_{z}-1: 0$ do

$8.1 Q \leftarrow 2 Q$.

8.2 For $j=1,2, z=1$ do

If $G_{z, j, i} \neq 0$ then:

If $G_{z, j, i}>0$ then $Q \leftarrow Q+k_{z, j, i} P_{j}$;

Else $Q \leftarrow Q-\left|k_{z, j, i}\right| P_{j}$.

Step 9. For $j=1,2, z=2$ do

If $G_{z, j, i} \neq 0$ and $s=1,3$ then

If $G_{z, j, i}>0$ then $Q \leftarrow Q+k_{z, j, i} P_{s}$;

Else $Q \leftarrow Q-\left|k_{z, j, i}\right| P_{s}$.

Step 10. Return $Q$.

\section{Appendix D. ISD Method to Compute Point Multiplication Elliptic Curve $k P$}

Algorithm 4 (ISD Method to Compute Point Multiplication Elliptic Curve $k P)$. This algorithm consists of the following steps:

Step 1. Apply GLV generator algorithm in [11] to find the generator $\left\{v_{1}, v_{2}\right\}$ for the given $n$ and $\lambda$ such that $v_{1} \leftarrow(r, t)$ and $v_{2} \leftarrow(u, v)$.

Step 2. Use balanced length-two representation of a multiplier algorithm in [3] to decompose $k$ to find $k_{1}$ and $k_{2}$ for a given $n, \lambda$ and $k \in[1, n-1]$.

As for the proposed steps for modification, they include the following:

Step 3. Use algorithm (2) to find

3.1 For $n$ and $\lambda_{1}$, generate the ISD generator $\left\{v_{3}, v_{4}\right\}$ such that $v_{3} \leftarrow(\bar{r}, \bar{t})$ and $v_{4} \leftarrow(\bar{u}, \bar{v})$.

3.2 For $n$ and $\lambda_{2}$, generate the ISD generator $\left\{v_{5}, v_{6}\right\}$ such that $v_{5} \leftarrow(\hat{r}, \hat{t})$ and $v_{6} \leftarrow(\hat{u}, \hat{v})$.

Step 4. Use algorithm (3) to decompose $k_{1}$ and $k_{2}$ such that $k_{1}=k_{11}+$ $k_{12} \lambda_{1}(\bmod n)$ and $k_{2}=k_{21}+k_{22} \lambda_{2}(\bmod n)$. That is, one can get $k=k_{11}+k_{12} \lambda_{1}+k_{21}+k_{22} \lambda_{2}(\bmod n)$. 
Step 5. Use algorithm (4) to compute $k P$ defined as

$$
\begin{aligned}
k P & =k_{11} P+k_{12}\left[\lambda_{1}\right] P+k_{21} P+k_{22}\left[\lambda_{2}\right] P \\
& =k_{11} P+k_{12} \psi_{1}(P)+k_{21} P+k_{22} \psi_{2}(P) .
\end{aligned}
$$

such that $\psi_{1}(P) \leftarrow\left[\lambda_{1}\right] P$ and $\psi_{2}(P) \leftarrow\left[\lambda_{2}\right] P$, where $\lambda_{1}, \lambda_{2} \in Z$ and $\lambda_{1} \neq \pm \lambda_{2}$. 